\title{
System Identification of Wood Drying Process Based on ARMAX Model
}

\author{
Zheng Zhou, Pinxiu Zhang*, Baofu Huai, Liping Huang \\ Electrical and Information College, Heilongjiang Bayi Agricultural University, Daqing, China \\ Email: *824363820@qq.com
}

How to cite this paper: Zhou, Z., Zhang, P.X., Huai, B.F. and Huang, L.P. (2019) System Identification of Wood Drying Process Based on ARMAX Model. Agricultural Sciences, 10, 241-248. https://doi.org/10.4236/as.2019.103020

Received: October 17, 2018

Accepted: March 8, 2019

Published: March 11, 2019

\begin{abstract}
This article presents system identification of wood drying process based on ARMAX model. Temperature and equivalent moisture content are considered as inputs, and moisture content of the wood sample during drying is taken as output of the system. The comparative study of RLS and FF-RLS to identify the system parameters is presented. Simulation results are presented to validate the efficacy of the ARMAX model for wood drying process.
\end{abstract}

\section{Keywords}

Wood Drying, System Identification, Moisture Content

\section{Introduction}

Wood drying process plays an important role in industry of wood product [1]. The quality of wood product mainly depends on the final moisture content of wood. Temperature and equilibrium moisture content (EMC) are the two main factors influencing the drying moisture content [2]. Hence, experimental data of temperature and EMC was often used to build the prediction model of wood drying moisture in the previous literature.

Wood drying prediction models like [3] [4] [5] [6], simplified physical characteristics are used to simulate the coupled heat and mass transfer during wood drying. However, too many inputs, e.g., gravity, external pressure, capillarity, temperature gradient, water concentration, etc., used to build theoretical models has a deterioration in accuracy to solve the highly coupled equations. Mathematical method such as artificial neural networks (ANN) and support vector machines (SVM) have been used to build drying model due to their ability to capture the trend of moisture content [7] [8] [9] [10]. ANN model can provide accurate prediction results, however over-training problem must to be taken 
care of to develop the prediction model. SVM model depends on a subset of training data points. Though incomplete data can be used to develop a SVM model, it also requires a substantial number of computation times to solve large-size equations [11].

Prediction models based on Auto Regressive Moving Average model with exogenous input (ARMAX) analysis have been widely shown in literature [12] [13] [14] [15] because of the computational efficiency. To the best of authors' knowledge, ARMAX model applied in the field of wood drying have rarely been seen in the previous research. In this paper, the method of ARMAX is presented to describe the time-varying system of wood drying. Recursive Least Squares (RLS) is introduced to identify the coefficients of the ARMAX model. To improve the identifying accuracy, an optimization algorithm is also discussed. Wood sample used in this study for the drying experiment was Northeast China ash. The dying kiln and its inside structure are show in Figure 1. The temperature, EMC, and moisture content used in the simulation model were the average value collected by temperature sensors, EMC sensors, and moisture content sensors.

\section{Mathematics Model Description}

The model takes temperature and EMC as inputs, moisture content as output. ARMAX model of wood drying process is described as

$$
A(z) y(t)=B_{1}(z) u_{1}(t)+B_{2}(z) u_{2}(t)+D(z) v(t)
$$

The constant coefficient polynomials $A(z), B_{1}(z), B_{2}(z)$ in the wood drying process model are

$$
\begin{gathered}
A(z)=a_{0}+a_{1} z^{-1}+a_{2} z^{-2} \\
B_{1}(z)=b_{10}+b_{1} z^{-1}+b_{2} z^{-2} \\
B_{2}(z)=b_{20}+b_{3} z^{-1}+b_{4} z^{-2} \\
D(z)=d_{0}+d_{1} z^{-1}
\end{gathered}
$$

When $t \leq 0, y(t)=0, \quad u_{j}(t)=0, v(t)=0, \quad a_{0}=1, \quad b_{10}=0, \quad b_{20}=0$, $d_{0}=0$.

Substituting polynomials (2), (3), (4), (5) and the initial values into (1), gives

$$
\begin{aligned}
& {\left[1+a_{1} z^{-1}+a_{2} z^{-2}\right] y(t)} \\
& =\left[b_{1} z^{-1}+b_{2} z^{-2}\right] u_{1}(t)+\left[b_{3} z^{-1}+b_{4} z^{-2}\right] u_{2}(t)+\left(d_{1} z^{-1}\right) v(t)
\end{aligned}
$$

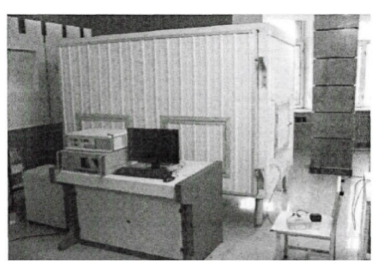

(a)

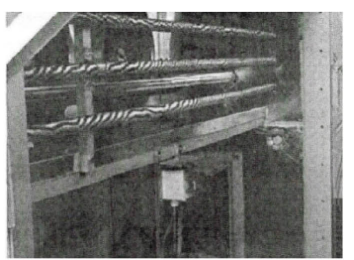

(b)

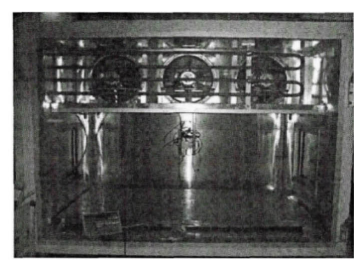

(c)

Figure 1. Wood drying kiln. (a) Wood drying kiln exterior; (b) Humidifying tube and heating tube; (c) Fans. 
Introducing the unit backward shift operator $z^{-1}$ into (6) yields to

$$
\begin{aligned}
y(t)= & -a_{1} y(t-1)-a_{2} y(t-2)+b_{1} u_{1}(t-1)+b_{2} u_{1}(t-2) \\
& +b_{3} u_{2}(t-1)+b_{4} u_{2}(t-2)+d_{1} v(t-1)
\end{aligned}
$$

Therefore, the output $y(t)$ can be expressed as

$$
y(t)=\varphi^{\mathrm{T}}(t) \theta+v(t)
$$

where $\theta:=\left[a_{1}, a_{2}, b_{1}, b_{2}, b_{3}, b_{4}, d_{1}\right]^{\mathrm{T}}, \varphi(t)=\left[-y(t-1),-y(t-2), u_{1}(t-1), u_{1}(t-\right.$ $\left.2), u_{2}(t-1), u_{2}(t-2), v(t-1)\right]^{\mathrm{T}}$.

\section{Parameter Identification}

Let $t=1,2, \cdots$, Equation (8) leads to

$$
Y_{t}=H_{t} \theta+V_{t}
$$

Using the Least Squares identification principle to define the quadratic criterion function

$$
J(\theta):=V_{t}^{\mathrm{T}} V_{t}=\left(Y_{t}-H_{t} \theta\right)^{\mathrm{T}}\left(Y_{t}-H_{t} \theta\right)
$$

Using recursive method, matrix $P^{-1}(t)$ is define as

$$
P^{-1}(t)=P^{-1}(0)+\sum_{j=1}^{t} \varphi(j) \varphi^{\mathrm{T}}(j)=P^{-1}(0)+H_{t}^{\mathrm{T}} H_{t}, P(0)=p_{0} I>0
$$

The RLS estimation of the parameter vector is

$$
\hat{\theta}(t)=\left(H_{t}^{\mathrm{T}} H_{t}\right)^{-1} H_{t}^{\mathrm{T}} Y_{t}=P(t) H_{t}^{\mathrm{T}} Y_{t}=\hat{\theta}(t-1)+P(t) \varphi^{\mathrm{T}}(t)\left[y(t)-\varphi^{\mathrm{T}}(t) \hat{\theta}(t-1)\right]
$$

The estimated residual is

$$
\hat{v}(t)=y(t)-\hat{\varphi}(t) \hat{\theta}(t)
$$

where $\hat{\varphi}(t):=\left[\phi(t)^{\mathrm{T}}, \hat{v}(t-1)\right]^{\mathrm{T}}$.

RLS estimation of parameter vector $\theta$ is achieved

$$
\begin{aligned}
& \hat{\theta}(t)=\hat{\theta}(t-1)+P(t) \hat{\varphi}(t)\left[y(t)-\hat{\varphi}^{\mathrm{T}}(t) \hat{\theta}(t-1)\right] \\
& P^{-1}(t)=P^{-1}(t-1)+\hat{\varphi}(t) \hat{\varphi}^{\mathrm{T}}(t), P(0)=p_{0} I>0
\end{aligned}
$$

In experimental system, data accumulates with time, results in the failure of extracting new data information from the previous data. Especially to the time-varying parameter system, due to the characteristics of parameter, the algorithm should track the time variation parameter. Hence, forgetting factor $\lambda$ is introduced into (15), an optimization algorithm to identify the parameters is obtained

$$
P^{-1}(t)=\lambda P^{-1}(t-1)+\hat{\varphi}(t) \hat{\varphi}^{\mathrm{T}}(t)
$$

\section{Simulation Results}

\subsection{Computation of the Model Parameters Based on RLS}

Simulation results were based on the 1000 input-output experimental data acquired during wood drying. RLS algorithm in Equations (14) and (15) is applied 
to estimate the parameters in the ARMAX model built in Equation (1). Parameters variation trend is shown in Figure 2. Comparison of actual values and predicted values are shown in Figure 3. Figure 4 is the absolute error between predicted value and actual value. Mean Square Error (MSE) and Root Mean Square Error (RMSE) of RLS algorithm is 0.2973 and 0.5387 , respectively.

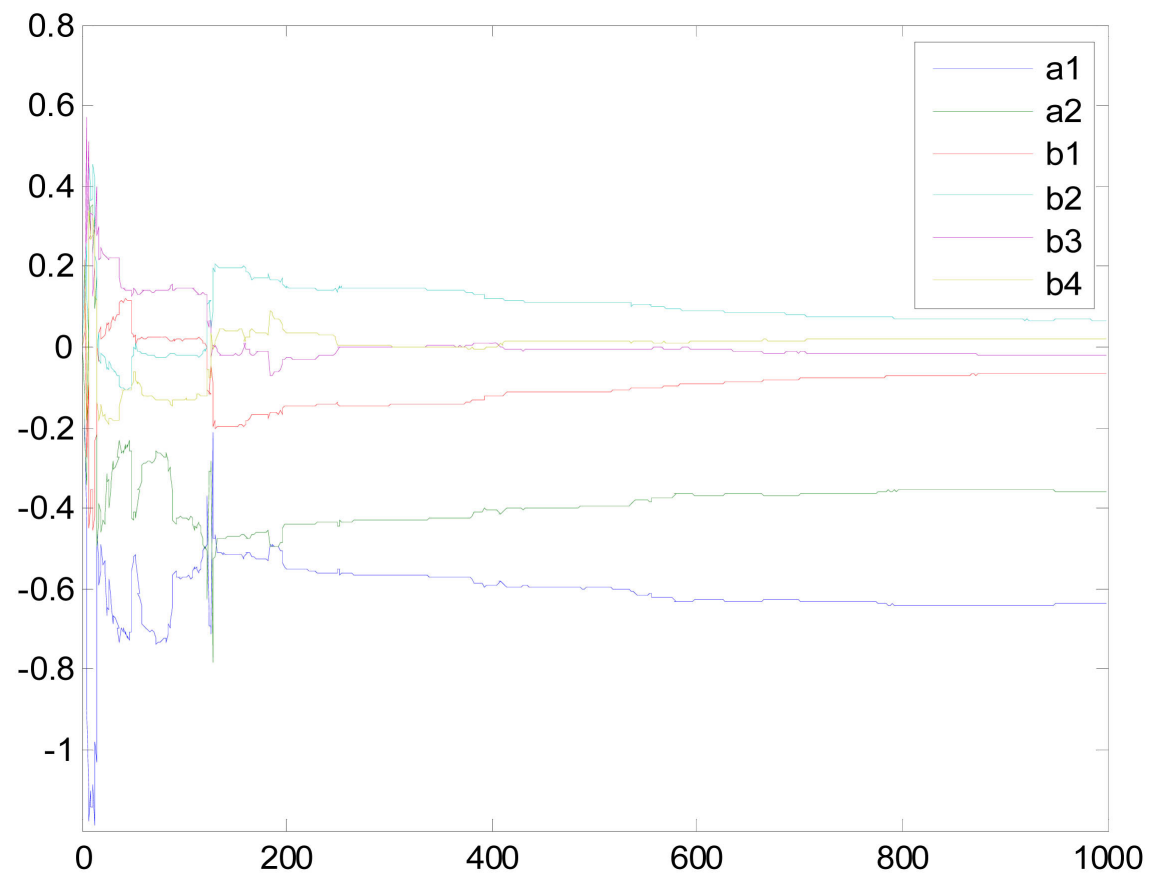

Figure 2. Estimate parameters $a_{1}, a_{2}, b_{1}, b_{2}, b_{3}, b_{4}, d_{1}$.

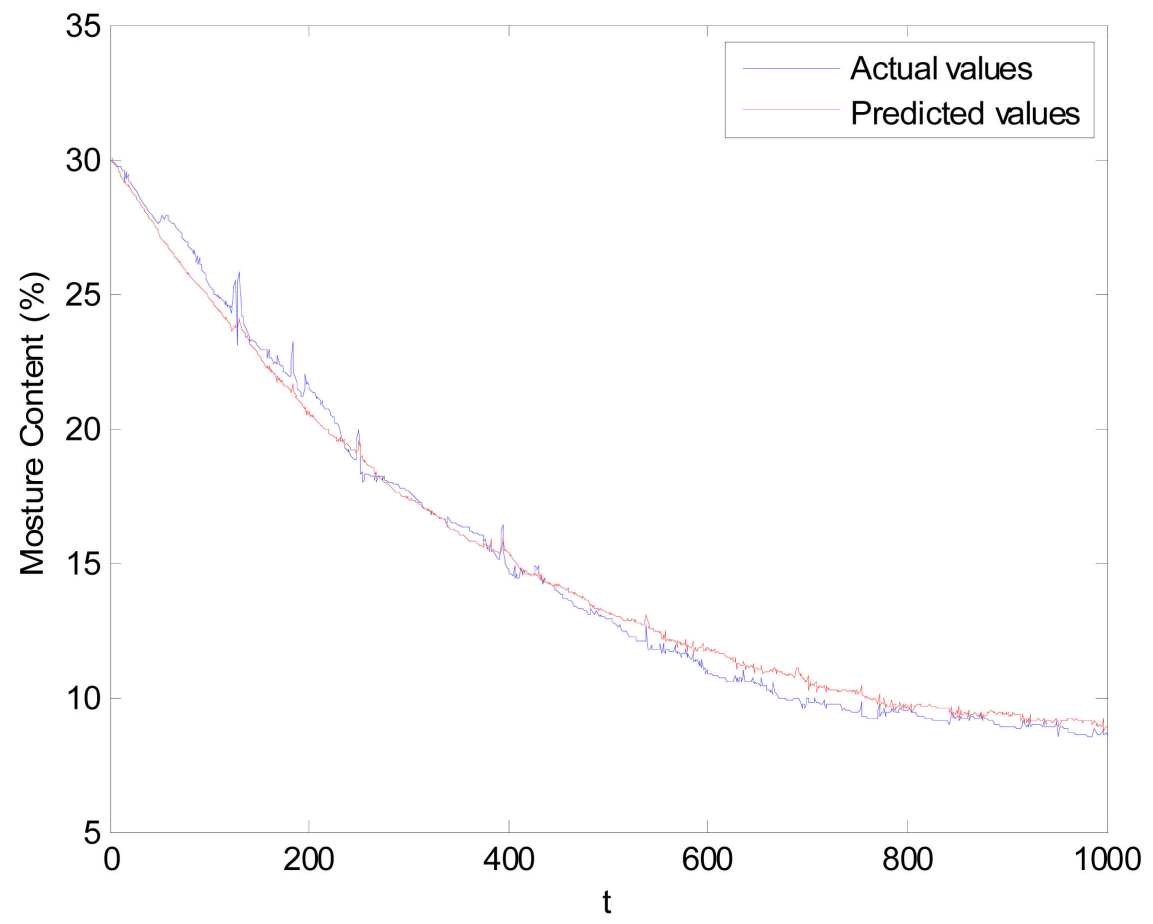

Figure 3. Comparison of actual values and predicted values. 


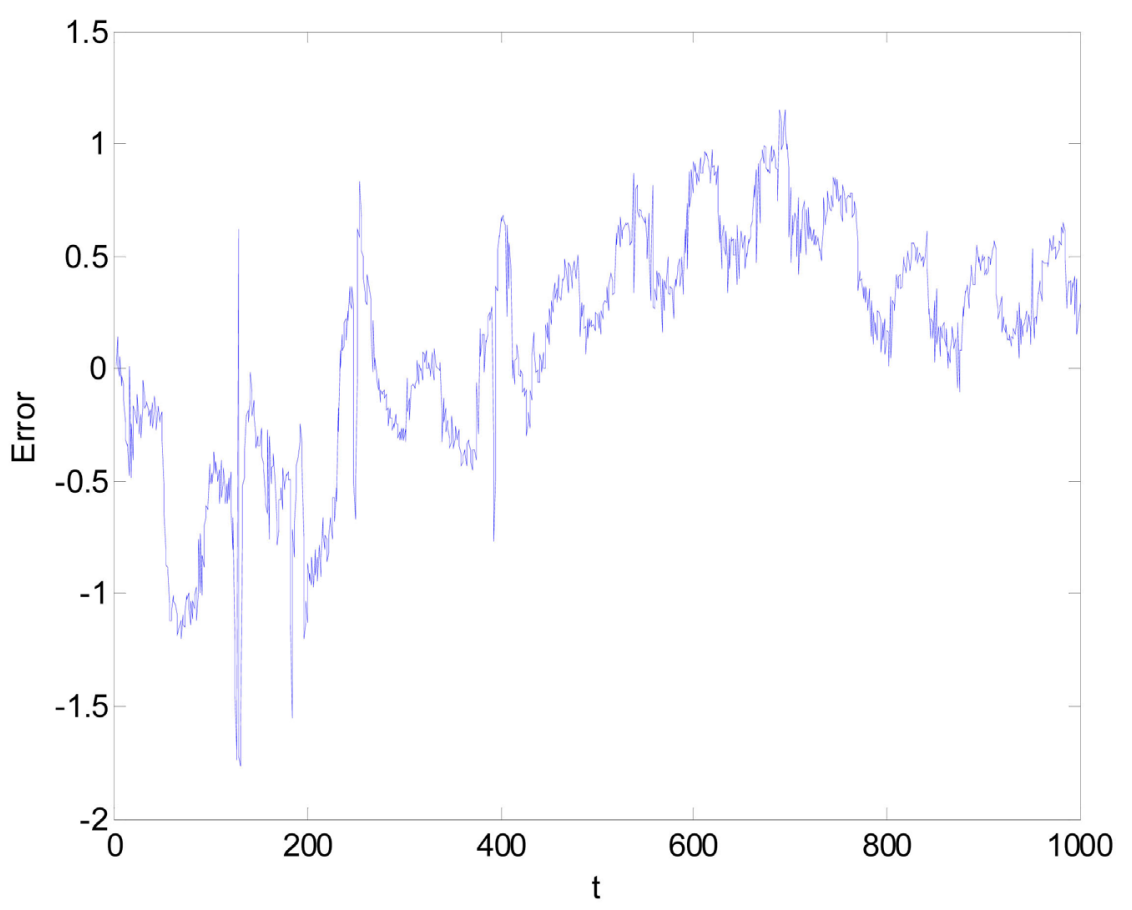

Figure 4. Absolute error.

\subsection{Computation of the Model Parameters Based on FF-RLS}

Parameters variation trend with $\lambda=0.95$ is shown in Figure 5. It is obviously observed that the FF-RLS (Forgetting Factor Recursive Least Squares) algorithm has a faster convergence speed than RLS algorithm. From $t=200$, curves of estimate parameters tend to be stable. The comparison of actual values and predicted values is shown in Figure 6. The absolute error between predicted value and actual values is shown in Figure 7. MSE is 0.3630, and RMSE is 0.7111. Convergence speed of FF-RLS algorithm is faster, however the absolute error is greater than RLS algorithm.

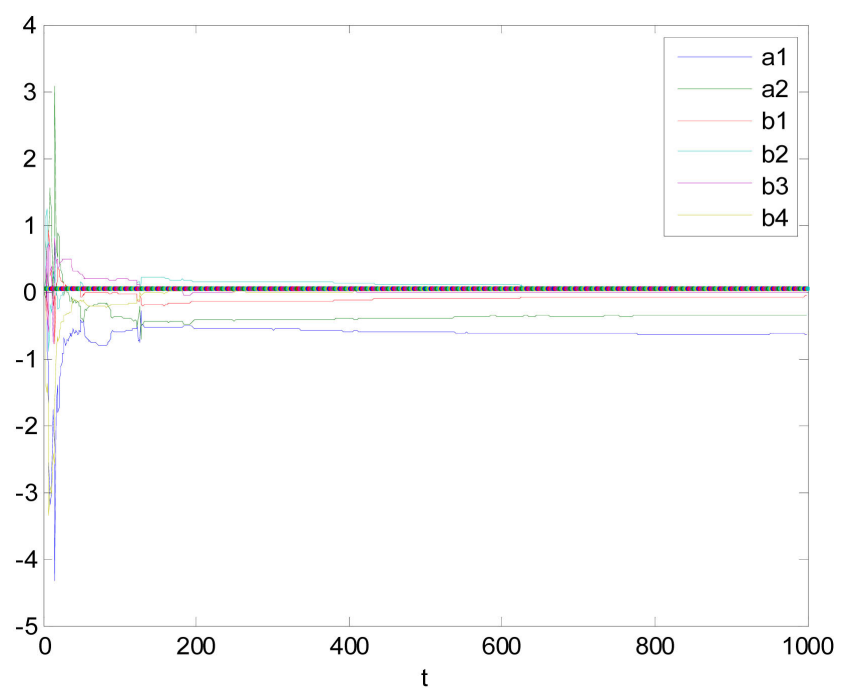

Figure 5. Estimate parameters $a_{1}, a_{2}, b_{1}, b_{2}, b_{3}, b_{4}, d_{1}$. 


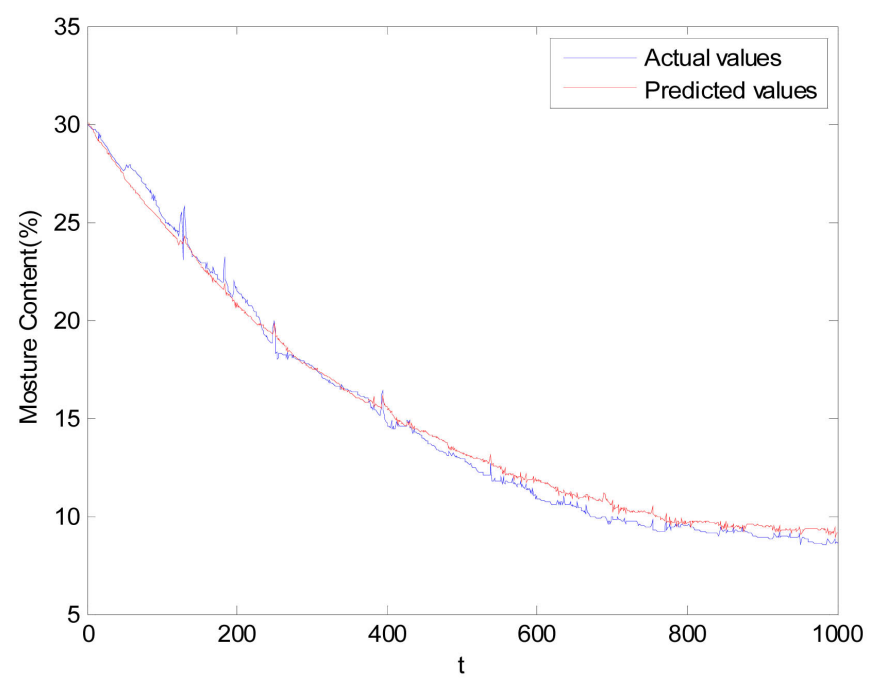

Figure 6. Comparison of actual values and predicted values.

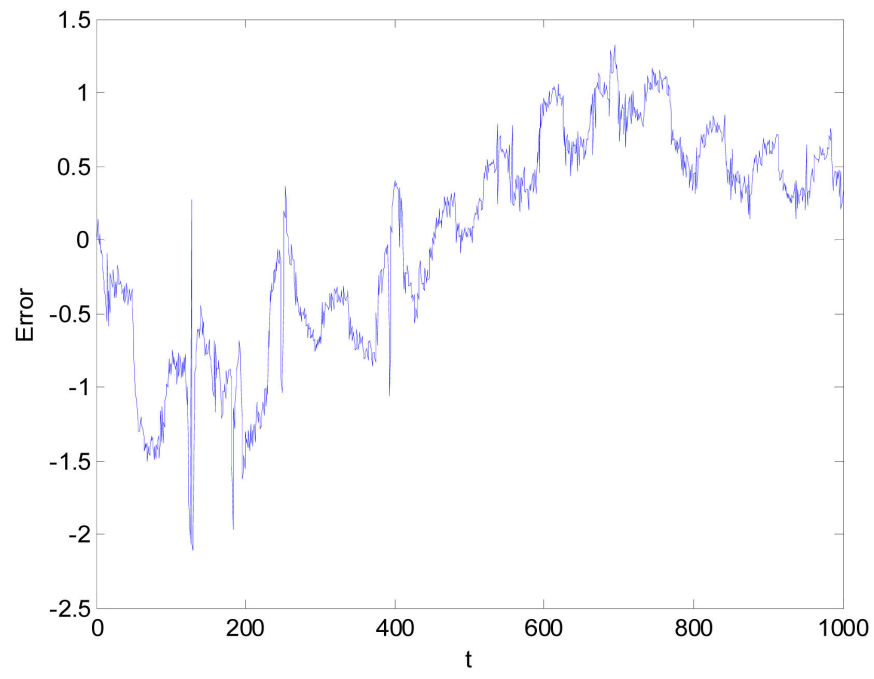

Figure 7. Absolute error.

\section{Conclusion}

In this paper, an ARMAX model based on the experimental data is derived to describe the wood drying model, which is adopted to predict wood moisture content during drying. RLS and FF-RLS algorithms are utilized to identify the system parameters. The proposed method is verified by simulation results. The parameters variation trend with the proposed prediction scheme is studied. Simulation results demonstrate that the FF-RLS method leads to a faster and more stable convergence compared with RLS scheme. However, the accuracy of RLS estimate is higher than FF-RLS.

\section{Acknowledgements}

This work was supported by University Nursing Program for Young Scholars with Creative Talents in Heilongjiang Province (UNPYSCT-2018083). 


\section{Conflicts of Interest}

The authors declare no conflicts of interest regarding the publication of this paper.

\section{References}

[1] Da Silva, W.P., et al. (2010) Optimization and Simulation of Drying Processes Using Diffusion Models: Application to Wood Drying Using Forced Air at Low Temperature. Wood Science and Technology, 45, 787-800.

https://doi.org/10.1007/s00226-010-0391-x

[2] Situmorang, Z. and Situmorang, J.A. (2016) Intelligent Fuzzy Controller for a Solar Energy Wood Dry Kiln Process. IEEE International Conference on Technology, Informatics, Management, Engineering and Environment, 152-157.

[3] Simo-Tagne, M., et al. (2016) Modeling of Coupled Heat and Mass Transfer during Drying of Tropical Woods. International Journal of Thermal Sciences, 109, 299-308. https://doi.org/10.1016/j.ijthermalsci.2016.06.012

[4] Scherer, V., et al. (2016) Coupled DEM-CFD Simulation of Drying Wood Chips in a Rotary Drum-Baffle Design and Model Reduction. Fuel, 184, 896-904. https://doi.org/10.1016/j.fuel.2016.05.054

[5] Zadin, V., et al. (2015) Application of Multiphysics and Multiscale Simulations to Optimize Industrial Wood Drying Kilns. Applied Mathematics and Computation, 267, 465-475. https://doi.org/10.1016/j.amc.2015.01.104

[6] Hasan, M. and Langrish, T.A.G. (2016) Time-Valued Net Energy Analysis of Solar Kilns for Wood Drying: A Solar Thermal Application. Energy, 96, 415-426. https://doi.org/10.1016/j.energy.2015.11.081

[7] Ge, L. and Chen, G.-S. (2014) Control Modeling of Ash Wood Drying Using Process Neural Networks. Optik-International Journal for Light and Electron Optics, 125, 6770-6774. https://doi.org/10.1016/j.ijleo.2014.07.091

[8] Nadian, M.H., et al. (2015) Continuous Real-Time Monitoring and Neural Network Modeling of Apple Slices Color Changes during Hot Air Drying. Food and Bioproducts Processing, 94, 263-274. https://doi.org/10.1016/j.fbp.2014.03.005

[9] Zhang, D., Cao, J. and Sun, L. (2010) Notice of Retraction Dynamic Modeling of Wood Drying Process Based on SLSSVM. IEEE International Conference on Computer Science and Information Technology, 1, 431-435.

[10] Shibata, H. and Hirohashi, Y. (2013) Effect of Segment Scale in a Pore Network of Porous Materials on Drying Periods. Drying Technology, 31, 743-751. https://doi.org/10.1080/07373937.2012.752742

[11] Dong, B., et al. (2005) Applying Support Vector Machines to Predict Building Energy Consumption in Tropical Region. Energy and Buildings, 37, 545-553. https://doi.org/10.1016/j.enbuild.2004.09.009

[12] Yin, L., Liu, S. and Gao, H. (2018) Regularised Estimation for Armax Process with Measurements Subject to Outliers. IET Control Theory and Applications, 12, 865-874. https://doi.org/10.1049/iet-cta.2017.1204

[13] Muhammad, A., et al. (2018) Forecasting of Global Solar Radiation Using Anfis and Armax Techniques. IOP Conference Series: Materials Science and Engineering, 303, 012016. https://doi.org/10.1088/1757-899X/303/1/012016

[14] Ratih, I.D., et al. (2018) Value-at-Risk Analysis Using ARMAX GARCHX Approach 
for Estimating Risk of Banking Subsector Stock Return's. Journal of Physics: Conference Series, 974, 012029. https://doi.org/10.1088/1742-6596/974/1/012029

[15] Jorge Barrientos Marín, E.T.O. and Velilla, E. (2018) Forecasting Electricity Price in Colombia: A Comparison between Neural Network, ARMA Process and Hybrid Models. International Journal of Energy Economics and Policy, 8, 97-106. 